\title{
Quality Improvement Interventions to Prevent Unplanned Extubations in Pediatric Critical Care: A Systematic Review
}

Krista Wollny ( $\sim$ krista.wollny@ucalgary.ca )

University of Calgary https://orcid.org/0000-0002-7709-1030

\section{Sara Cui}

University of Calgary

Deborah McNeil

University of Calgary

Karen Benzies

University of Calgary

Simon J. Parsons

Alberta Children's Hospital

\section{Tolulope Sajobi}

University of Calgary

Amy Metcalfe

University of Calgary

\section{Research Article}

Keywords: Intensive Care Units, Pediatric, Patient Safety, Systematic Review, Extubation, Quality Improvement, Critical Care

Posted Date: January 18th, 2022

DOI: https://doi.org/10.21203/rs.3.rs-1262101/v1

License: (a) (i) This work is licensed under a Creative Commons Attribution 4.0 International License. Read Full License 


\section{Abstract}

\section{Background}

An unplanned extubation is the uncontrolled and accidental removal of a breathing tube. The health impact of an unplanned extubation ranges from minimal to life-threatening, but this is an important quality indicator in pediatric critical care.

\section{Objective}

To comprehensively synthesize literature published on quality improvement (QI) practices implemented to reduce the rate of unplanned extubations in critically ill children.

\section{Methods}

Data Sources and Study Selection: A search was conducted in MEDLINE, Embase, and CINHAL from inception through April 29, 2021. Two reviewers independently screened citations in duplicate using predetermined eligibility criteria.

Data Extraction and Data Synthesis: Data from included studies were abstracted using a tool created by the authors, and QI interventions were categorized using the Behavior Change Wheel. Study quality was assessed using the Quality Improvement Minimum Quality Criteria Set (QI-MQCS). Results were presented as descriptive statistics and narrative syntheses.

\section{Results}

Twelve studies were included in the final review. Ten described primary QI projects; two were sustainability studies that followed up on previously described QI interventions. Half of the included studies were rated as high-quality. The median number of QI interventions described by each study was 4.5 [IQR 4-5], with a focus on guidelines, environmental restructuring, education, training, and communication. Nine studies reported decreased unplanned extubation rates after the QI intervention; of these, six had statistically significant reductions. Both sustainability studies observed increased rates that were not statistically significant. 
This review provides a comprehensive synthesis of QI interventions to reduce unplanned extubation. With only half the studies achieving a high-quality rating, there is room for improvement when conducting and reporting research in this area. Findings from this review can be used to support clinical recommendations to prevent unplanned extubations, and support patient safety in pediatric critical care.

\section{Systematic Review Registration}

This review was registered on PROSPERO (CRD42021252233) prior to data extraction.

\section{Introduction}

Endotracheal intubation is a life-saving intervention in the Pediatric Intensive Care Unit (PICU), used to assist children with breathing when they are unable to do so on their own. An unplanned extubation is defined as the uncontrolled and accidental removal of the endotracheal tube (ETT) and can adversely affect health outcomes (1-3). Unplanned extubations are associated with increased number of ventilation days, longer length of stay in both the PICU and the hospital, increased medical expenses, and increased morbidity and mortality. Risk factors for unplanned extubation in the PICU include younger age (4-7), agitation (8-10), oral intubation (5), poor ETT fixation (11-13), copious secretions $(4,7,14)$, patient procedures $(15,16)$, inadequate sedation $(17,18)$, repositioning/ transport $(10,19)$, lack of restraints $(4,14,20)$, and nurse-patient ratios $(9,14)$.

Recent studies have demonstrated rates of unplanned extubation in PICUs range between 0.11 to 6.4 events/100 airway days (1-3). Quality improvement (QI) projects have been effective in decreasing the rate of unplanned extubations, usually targeting a rate less than $0.6 / 100$ airway days $(13,21,22)$. Quality improvement has been defined as "systematic, data-guided activities designed to bring about immediate, positive changes in the delivery of health care in particular settings"(23). The purpose of quality improvement is to deliver healthcare that is safe, effective, patient-centred, timely, efficient and equitable (24). Quality improvement involves continuous changes to practice at the local level, with the aim to improve patient and population health outcomes. Quality improvement interventions to prevent unplanned extubations have been primarily targeted towards staff education, standardizing sedation protocols, and standardizing procedures such as ETT, hygiene, and transport $(10,21,22)$. Due to the varying patient care practices in individual PICUs, no single prevention procedure is followed by all PICUs.

The purpose of this article is to comprehensively review the literature that has been published on QI practices implemented to reduce the rate of unplanned extubations in critically ill children. Findings from this review will support clinical recommendations to prevent unplanned extubations, positively supporting patient safety in pediatric critical care.

\section{Methods}


This systematic review was conducted and reported according to the Preferred Reporting Items for Systematic Review and Meta-analyses (PRISMA) 2020 reporting guidelines (25) (Supplemental Table 1) and was registered on PROSPERO (CRD42021252233) prior to data extraction.

\section{Data Sources and Searches}

Literature searches were conducted in MEDLINE, Embase and CINAHL, from database inception to April 29,2021 . The search strategies for each database were developed using literature on the topic and in consultation with clinical experts. The search strategies were revised after reviewing preliminary search results, and included synonyms for 1 ) pediatric intensive care; 2) quality improvement; and 3) unplanned extubations. No limitations on date or language were used for the search. The complete MEDLINE search strategy is shown in Supplemental Table 2. Citation searching of included articles was also used to handsearch for relevant literature. References were managed and de-duplicated in Covidence (Covidence systematic review software, Veritas Health Innovation, Melbourne, Australia).

\section{Eligibility Criteria}

Inclusion criteria consisted of original, primary research on quality improvement interventions to reduce the rate of unplanned extubations in pediatric critical care. Studies were excluded if they 1 ) were not primary research (e.g., reviews, commentaries); 2) had a population focus outside of pediatric critical care (e.g., adult critical care or neonatal critical care); 3) did not describe QI interventions to reduce the rate of unplanned extubations; 4) did not include unplanned extubation rates; 5 ) were only presented as an abstract; or 6) were not published in English language.

\section{Study Selection}

After a subset of the team (KW, SC) achieved $100 \%$ agreement on a random sample of 10 citations, all titles and abstracts were reviewed independently and in duplicate by two reviewers (KW, SC). Any discrepancies were resolved by discussion. The full-text articles were then reviewed independently and in duplicate by two reviewers (KW, SC); again, any discrepancies were resolved by discussion, or the involvement of a third reviewer (AM) when necessary.

\section{Data Abstraction and Quality Assessment}

Two reviewers (KW, SC) independently abstracted and reviewed data for each included study using a data abstraction sheet developed by the review team. Discrepancies were resolved through discussion with a third reviewer (AM). Information on study characteristics (e.g., year of publication, country, ICU type), QI intervention characteristics (e.g., description of interventions, time frame, barriers and facilitators) and outcomes (e.g., rates of unplanned extubation, statistical significance) were collected.

The Quality Improvement Minimum Quality Criteria Set (QI-MQCS) was used to assess the reporting of the quality improvement evaluation studies (26). The QI-MQCS includes 16 domains, all of which were assessed at the study-level. Similar to other studies in this area, studies that report $\geq 14$ domains are considered high quality (27). 


\section{Data Synthesis and Analysis}

Findings were analyzed and presented as a narrative synthesis. All descriptive analyses were performed using STATA 15 software (StataCorp. 2017. Stata Statistical Software: Release 15. College Station, TX: StataCorp LLC). Due to heterogeneity between studies, and the variable nature of QI interventions, metaanalysis was not performed.

Quality improvement interventions were categorized using the Behavior Change Wheel (BCW) (28). The $\mathrm{BCW}$ is a model that was developed as a comprehensive synthesis of several other behavior change frameworks. It centers on the COM-B system, which recognizes that behavior change stems from capability, opportunity and motivation (28). Surrounding the COM-B system are intervention functions, which are then encompassed by policy categories. This acknowledges that policies (e.g., guidelines, regulations) can influence behavior through interventions (e.g., enablement, environmental restructuring) (28). The described QI interventions within each study were coded as intervention functions (e.g., education, enablement, modeling) and/or policy categories (e.g., guidelines, regulations) (28). A QI intervention as described by authors could fall into more than one category. Coding was done independently and in duplicate; any discrepancies were resolved through discussion.

\section{Results}

\section{Results of the Search}

The search strategy identified 128 records. After duplicates were removed, we screened 87 unique abstracts and reviewed 36 full-text articles; 27 full-text articles were excluded, the most common reasons being abstract-only publications, not describing quality improvement interventions, or not English language. See PRISMA flow diagram (Figure 1). Citation searching identified 3 potential studies, all of which were included. The frequency of disagreements and agreements between reviewers for the full-text screen was calculated at 0.880 using Cohen's Kappa statistic.

\section{Included Studies}

Twelve studies were included in the review. Study characteristics are summarized in Table 1 and Supplemental Table 3. The studies were published between 2004-2020, and took place in the United Sates $(n=9 ; 75.0 \%)$, Canada $(n=2 ; 16.7 \%)$ and Brazil $(n=1 ; 8.3 \%)$. Most studies were conducted in a PICU $(n=8 ; 66.7 \%)$; three studies took place in both a PICU and a Cardiac Intensive Care Unit (CICU), and one took place in a CICU only. Reported unit sizes ranged from 5-26 beds per unit. Eleven of the 12 studies took place at a single center; one was a multi-center study that included PICUs and CICUs from 43 children's hospitals (21). The majority were prospective cohort studies ( $n=9 ; 75.0 \%)$; two studies had retrospective cohort designs, and one was mixed-methods. 


\section{Quality Improvement Initiatives}

Two studies $(13,17)$ were designed to assess the sustainability of quality improvement initiatives, and were analyzed separately from the other 10 primary studies. Both assessed the sustainability of QI studies included in this review $(29,30)$.

Studies were conducted over a period of 12-110 months, which, for most, included pre- and postimplementation data collection. Of the studies that explicitly reported the implementation period, interventions were implemented over a range of 3-24 months. Rates of unplanned extubation were collected up to 6-48 months post-intervention. QI interventions are detailed in Supplemental Table 3.

The median number of interventions implemented in each study was 4.5 [IQR: 4-5] (Table 2). Most intervention functions implemented were categorized as environmental restructuring (e.g., standardizing practice through a guideline, protocol, or algorithm; $n=9$ ) or education (e.g., workshops, education days; $n=7)$. Most of the policy categories were classified as guidelines (e.g., sedation algorithms or tube securement protocols; $n=9$ ) and communication/ marketing (e.g., posters, e-mails; $n=7$ ). Guidelines were mainly focused on sedation practices $(n=5)$, standardization of care for intubated patients $(n=5)$, and ETT fixation/ securement $(n=4)$; many sites implemented more than one guideline as part of the QI project $(n=7)$.

Reported barriers to implementation included decreased baseline uniformity of practice, nurses being pulled away from the bedside, and frequent rotation of staff (Supplemental Table 3). Facilitators included increased nursing autonomy, culture change focused on safety, improved multi-disciplinary communication, and team leadership.

\section{Unplanned Extubation Rates}

All studies reported pre- and post-QI intervention unplanned extubation rates. Pre-intervention rates ranged from 0.44-6.40 events/100 airway days. Post-intervention rates ranged from 0-2.59 events/100 airway days. Nine studies reported a decreased rate of unplanned extubations after the QI intervention (Table 2). Of these, six studies reported a statistically significant $(p<0.05)$ decrease; three studies did not describe statistical significance. Two studies observed statistically significant decreases in one unit, but not in the other (e.g., PICU vs CICU). $(21,30)$

\section{Sustainability Studies}

Two studies reported the sustainability of QI interventions to prevent unplanned extubations. Rachman at al. (17) reported the rates of unplanned extubation nine years after they implemented QI interventions (29). Similarly, Censoplano et al. (13) studied the rates of unplanned extubations seven years after QI interventions were implemented (30). Both sustainability studies observed an increase in the rate of unplanned extubations ( 0 to 0.4 and 1 to $1.5 / 100$ airway days, respectively), however, these increases were not statistically significant. 


\section{Quality Assessment}

The median quality score using the QI-MQCS was 13.5 (IQR: 13-16). Five of the ten primary studies were rated high quality (Supplemental Table 4). Many of the domains were reported by all studies, including: organizational motivation, intervention rationale, intervention description, study design, comparator, data source, timing, health outcomes, and limitations. The domains that were reported the least include adherence/fidelity, sustainability and spread.

\section{Discussion}

This systematic review and narrative synthesis of quality improvement initiatives to prevent unplanned extubations in pediatric critical care identified interventions that have been used to change behavior, including education, environmental restructuring, guidelines, and communication/marketing. Most studies observed a decrease in unplanned extubation rates after the implementation of QI interventions, and two sustainability studies found that the decreased rate remained stable years later.

Most studies described several QI interventions implemented together to change healthcare provider behavior. Often, adverse events and patient safety issues, such as unplanned extubations, have multifactorial causes $(1,21,31)$. Previous work in the area of pediatric patient safety has outlined an approach to patient safety which includes 1 ) identifying the epidemiology of events/errors; 2 ) integrating a culture of patient safety; and 3) creating and implementing patient safety solutions (32). This multipronged implementation approach was observed in the studies included in this review. Guidelines or protocols often change behavior through environmental restructuring, which was observed as a frequently used behavior change technique. Marketing and communication, alongside education and training were also frequently implemented, targeting patient safety solutions to change. A similar review of QI interventions to reduce the rate of unplanned extubations in adult critical care also found most QI programs involved multiple interventions, including standardization of procedures (guidelines), and education (2). The guidelines and procedures were also targeting similar foci, including sedation, nurseto-patient ratios, and endotracheal tube fixation (2). The policy categories and intervention functions that were not identified in this review include incentivization, coercion, restrictions, legislation, service provision or fiscal measures. A similar review in critical care quality improvement also identified these as interventions that are less frequently used to enact change in this setting (33).

The included multi-site study by Klugman et al. (21) described a nationwide quality improvement initiative that included 43 sites across the United States, led by the Children's Hospitals' Solutions for Patient Safety (SPS) - a network dedicated to eliminate serious harm in pediatric care. $(34,35)$ An "Unplanned Extubation Quality Improvement Bundle" was developed, and sites were encouraged to implement the bundle locally using the Model for Improvement or Lean Six Sigma (21). The bundle - a set of evidence-based practice suggestions - standardized care, but allowed for site-specific contextual factors and preferences. Overall, the hospitals with higher bundle compliance had greater reduction in the 
rates of unplanned extubations. Bundles to reduce the rate of unplanned extubations have been effective in other patient populations, such as in neonatal intensive care (36).

Only half of the studies were rated as high-quality using the QI-MQCS (26). The domains that were reported the least were adherence/fidelity, sustainability and spread. Adherence/fidelity refers to the process measures within a QI study, such as how many learners attended an education session, or how often healthcare providers followed the guideline (26); process measures allow for interpretation about how well an intervention was implemented as planned (37). This lowers the quality of a QI study by omitting important contextual details that prevent comparisons between studies and reproducibility. Sustainability refers to the potential for intervention maintenance (26). A discussion about sustainability is important for understanding whether the outcome will persist, as long-term sustainability is the goal. Finally, spread refers to the ability of the intervention to be replicated in other settings (26). This concept is essential for clinicians to be able to conceptualize how the intervention could be implemented at their own site, considering contextual factors are key to an intervention's success. Overlooking the concept of spread may limit future generalizability.

The Standards for Quality Improvement Reporting Excellence (SQUIRE) guidelines were first published in 2008, and then revised in 2016 (38). Although most studies were published after the SQUIRE guidelines were released, none of the primary QI studies referenced the guidelines, and only one sustainability study did (17). Use of the SQUIRE guidelines in conducting and reporting future research will increase study quality and transparency.

This systematic review is not without limitations. Due to the heterogenous nature of QI interventions and their focus on local causes, facilitators, and barriers to change, we were unable to meta-analyze the results. To allow readers to interpret each QI study within the context of the local environment, we presented background information such as unit size and type, location, QI interventions, timeframes, and interpreted barriers and facilitators to change (Supplemental Table 3).

We did not include preliminary findings from conference proceedings, as not enough contextual information would be presented for analysis in an abstract. This may have limited the information located on the topic. We also only included studies published in English language, which may have excluded relevant studies. Furthermore, there is risk of publication bias: the increased likelihood of publication with significant results (39). We found and included one study that did not demonstrate decreased rates of unplanned extubations after implementing QI interventions (12); however, all other studies demonstrated the intended decrease in unplanned extubation rates, suggesting potential publication bias. Including more studies with null findings would allow for a comparison between interventions that did and did decrease the rates of unplanned extubations in pediatric critical care.

This systematic review has several strengths. Using the QI-MQCS, we were able to comprehensively assess the quality of the included studies using a validated evaluation tool specific to QI research (26). This also highlights where future literature on the topic could improve. Additionally, this is the first study to systematically synthesize the literature available on QI interventions to reduce the rate of unplanned 
extubations in pediatric critical care. This systematic review adds to the literature by providing a comprehensive overview of the QI interventions to decrease rates of unplanned extubations in pediatric critical care. Patient safety is an ongoing strategic priority at most children's hospitals, with unplanned extubations listed as a key quality indicator $(34,40,41)$. Future research in this area should continue to explore the sustainability of QI interventions to decrease the rate of unplanned extubations in pediatric critical care, to be able to observe if improved rates are maintained over time.

\section{Conclusions}

This is the first systematic review to narratively describe QI interventions to decrease the rate of unplanned extubations in pediatric critical care. Most studies described several interventions used together to decrease the rate of unplanned extubation, with the most frequent being guidelines, environmental restructuring, education, training and communication. The findings from this review can be used by other hospitals aiming to reduce their unplanned extubation rates; other pediatric critical care units can use this information to design and implement local QI interventions based on the contextual factors at their own sites.

\section{Declarations}

\section{Declarations}

\section{Ethics approval:}

Not applicable. All work was developed using published data.

\section{Consent for publication:}

Not applicable.

\section{Declarations}

\section{Competing interests:}

The authors declare that they have no competing interests.

\section{Funding:}

This work was supported by an Alberta Children's Hospital Research Institute Graduate Student Award (KW) and an Alberta Health Services Critical Care Strategic Clinical Network Summer Studentship (SC). The funders had no role in study design, data collection and analysis, decision to publish, or preparation of the manuscript. 


\section{Authors' contributions:}

$\mathrm{KW}, \mathrm{SC}$ and $\mathrm{AM}$ were the core team leading the systematic review. KW, SC and AM identified and selected the studies. KW and SC collected the data. KW, SC and AM analyzed the data. KW, SC and AM assessed the certainty of the evidence. KW, SC, DM, KB, SP, TS and AM provided advice as different stages. KW, SC, $\mathrm{DM}, \mathrm{KB}, \mathrm{SP}, \mathrm{TS}$ and $\mathrm{AM}$ approved the final version of the manuscript. $\mathrm{KW}$ is the guarantor and attests that all listed authors meet authorship criteria and that no others meeting the criteria have been omitted.

\section{Acknowledgements:}

Not applicable.

\section{References}

1. Al-Abdwani R, Williams CB, Dunn C, Macartney J, Wollny K, Frndova H, et al. Incidence, outcomes and outcome prediction of unplanned extubation in critically ill children: An 11year experience. J Crit Care. 2018;44:368-75.

2. da Silva PSL, Fonseca MCM. Unplanned Endotracheal Extubations in the Intensive Care Unit: Systematic Review, Critical Appraisal, and Evidence-Based Recommendations. Anesthesia \& Analgesia. 2012 May;114(5):pp. 1003-14.

3. da Silva PSL, Farah D, Fonseca MCM. Revisiting unplanned extubation in the pediatric intensive care unit: What's new? Heart Lung. 2017;46(6):444-51.

4. Fitzgerald RK, Davis AT, Hanson SJ. National Association of Children's Hospitals and Related Institution PICU Focus Group Investigators. Multicenter Analysis of the Factors Associated With Unplanned Extubation in the PICU. Blanton R BM Christensen M, Giaccone MJ, Grappe S, Green J, Lafond P, Layman A, Lostocco L, Montgomery V, Schmidt DR, Shambaugh K, Sourbeer M, Welch T, editor. Pediatr Crit Care Med. 2015;16(7):e217-23.

5. Kanthimathinathan HK, Durward A, Nyman A, Murdoch IA, Tibby SM. Unplanned extubation in a paediatric intensive care unit: prospective cohort study. Intensive Care Med. 2015;41(7):1299-306.

6. da Silva PSL, de Aguiar VE, Neto HM, de Carvalho WB. Unplanned extubation in a paediatric intensive care unit: impact of a quality improvement programme. Anaesthesia. 2008;63(11):1209-16.

7. Razavi SS, Nejad RA, Mohajerani SA, Talebian M. Risk factors of unplanned extubation in pediatric intensive care unit. Tanaffos. 2013;12(3):11-6.

8. Scott PH, Eigen H, Moye LA, Georgitis J, Laughlin JJ. Predictability and consequences of spontaneous extubation in a pediatric ICU: Critical Care Medicine. 1985 Apr;13(4):228-32.

9. Marcin JP, Rutan E, Rapetti PM, Brown JP, Rahnamayi R, Pretzlaff RK. Nurse staffing and unplanned extubation in the pediatric intensive care unit. Pediatr Crit Care Med. 2005;6(3):254-7. 
10. Tripathi S, Nunez DJ, Katyal C, Ushay HM. Plan to Have No Unplanned: A Collaborative, HospitalBased Quality-Improvement Project to Reduce the Rate of Unplanned Extubations in the Pediatric ICU. Respir Care. 2015;60(8):1105-12.

11. Meregalli CN, Jorro Baron FA, D'Alessandro MA, Danzi EP, Debaisi GE. Impact of a quality improvement intervention on the incidence of unplanned extubations in a Pediatric Intensive Care Unit. Arch Argent Pediatr. 2013;111(5):391-7.

12. Menon K, Dundon B, Twolan B-L, AlShammari S. Approach to unplanned extubations in a pediatric intensive care unit. Can J Crit Care Nurs. 2015;26(3):25-9.

13. Censoplano NM, Barrett CS, Ing RJ, Reichert K, Rannie M, Kaufman J. Achieving Sustainability in Reducing Unplanned Extubations in a Pediatric Cardiac ICU*: Pediatric Critical Care Medicine. 2020 Apr;21(4):350-6.

14. Ream RS, Mackey K, Leet T, Green MC, Andreone TL, Loftis LL, et al. Association of nursing workload and unplanned extubations in a pediatric intensive care unit. Pediatr Crit Care Med. 2007;8(4):36671.

15. Little LA, Koenig JCJ, Newth CJ. Factors affecting accidental extubations in neonatal and pediatric intensive care patients. Crit Care Med. 1990;18(2):163-5.

16. Piva J, Amantea S, Luchese S, Guigno K, Maia T, Finloft L. Accidental extubation in a pediatric intensive care unit. J Pedatr Rio J. 1995;2(71):72-6.

17. Rachman BR, Mink RB. A prospective observational quality improvement study of the sustained effects of a program to reduce unplanned extubations in a pediatric intensive care unit: A prospective observational quality improvement study. Pediatric Anesthesia. 2013 Jul;23(7):614-20.

18. Popernack ML, Thomas NJ, Lucking SE. Decreasing unplanned extubations: utilization of the Penn State Children's Hospital Sedation Algorithm. Pediatr Crit Care Med. 2004;5(1):58-62.

19. Frank BS, Lewis RJ. Experience with intubated patients does not affect the accidental extubation rate in pediatric intensive care units and intensive care nurseries. Pediatr Pulmonol. 1997;23(6):424-8.

20. Sadowski R, Dechert RE, Bandy KP, Juno J, Bhatt-Mehta V, Custer JR, et al. Continuous quality improvement: reducing unplanned extubations in a pediatric intensive care unit. Pediatrics. 2004;114(3):628-32.

21. Klugman D, Melton K, Maynord PO, Dawson A, Madhavan G, Montgomery VL, et al. Assessment of an Unplanned Extubation Bundle to Reduce Unplanned Extubations in Critically III Neonates, Infants, and Children. JAMA Pediatr. 2020 Jun 1;174(6):e200268.

22. Kandil SB, Emerson BL, Hooper M, Ciaburri R, Bruno CJ, Cummins N, et al. Reducing Unplanned Extubations Across a Children's Hospital Using Quality Improvement Methods. Pediatric Quality Safety. 2018;3(6):e114.

23. Margolis P, Provost LP, Schoettker PJ, Britto MT. Quality Improvement, Clinical Research, and Quality Improvement Research-Opportunities for Integration. Pediatric Clinics of North America. 2009 Aug;56(4):831-41. 
24. Institute of Medicine (US) Committee on Quality of Health Care in America. Crossing the Quality Chasm: A New Health System for the 21st Century [Internet]. Washington (DC): National Academies Press (US); 2001 [cited 2019 Dec 18]. Available from: http://www.ncbi.nlm.nih.gov/books/NBK222274/.

25. Page MJ, McKenzie JE, Bossuyt PM, Boutron I, Hoffmann TC, Mulrow CD, et al. The PRISMA 2020 statement: an updated guideline for reporting systematic reviews. BMJ. 2021 Mar 29;n71.

26. Hempel S, Shekelle PG, Liu JL, Sherwood Danz M, Foy R, Lim Y-W, et al. Development of the Quality Improvement Minimum Quality Criteria Set (QI-MQCS): a tool for critical appraisal of quality improvement intervention publications. BMJ Qual Saf. 2015 Dec;24(12):796-804.

27. Inata Y, Nakagami-Yamaguchi E, Ogawa Y, Hatachi T, Takeuchi M. Quality Assessment of the Literature on Quality Improvement in PICUs: A Systematic Review. Pediatric Critical Care Medicine [Internet]. 2021 Feb 26 [cited 2021 May 31];Publish Ahead of Print. Available from: https://journals.Iww.com/10.1097/PCC.0000000000002683.

28. Michie S, van Stralen MM, West R. The behaviour change wheel: A new method for characterising and designing behaviour change interventions. Implementation Sci. 2011 Dec;6(1):42.

29. Rachman BR, Watson R, Woods N, Mink RB. Reducing Unplanned Extubations in a Pediatric Intensive Care Unit: A Systematic Approach. International Journal of Pediatrics. 2009;2009:1-5.

30. Kaufman J, Rannie M, Kahn MG, Vitaska M, Wathen B, Peyton C, et al. An Interdisciplinary Initiative to Reduce Unplanned Extubations in Pediatric Critical Care Units. PEDIATRICS. 2012 Jun 1;129(6):e1594-600.

31. da Silva PSL, Fonseca MCM. Factors Associated With Unplanned Extubation in Children: A CaseControl Study. J Intensive Care Med. 2020 Jan;35(1):74-81.

32. Mueller BU, Neuspiel DR, Fisher ERS, COUNCIL ON QUALITY IMPROVEMENT AND PATIENT SAFETY, COMMITTEE ON HOSPITAL CARE. Principles of Pediatric Patient Safety: Reducing Harm Due to Medical Care. Pediatrics. 2019 Feb;143(2):e20183649.

33. Jarzenski T, Becker C, King E, Cooper S, Montague C, Mulhausen H, et al. Behavior Change Strategies used to Implement Early Mobility Programs in the Intensive Care Unit: A Systematic Review. 2019 Dec 8.

34. Lyren A, Brilli RJ, Zieker K, Marino M, Muething S, Sharek PJ. Children's Hospitals' Solutions for Patient Safety Collaborative Impact on Hospital-Acquired Harm. Pediatrics. 2017 Sep;140(3):e20163494.

35. Solutions for Patient Safety [Internet]. Available from: https://www.solutionsforpatientsafety.org/.

36. Merkel L, Beers K, Lewis MM, Stauffer J, Mujsce DJ, Kresch MJ. Reducing unplanned extubations in the NICU. Pediatrics. 2014;133(5):e1367-72.

37. Straus SE, Tetroe J, Graham ID, editors. Knowledge translation in health care: moving from evidence to practice. 2nd ed. Chichester: Wiley/BMJ Books; 2013. 406 p.

38. Ogrinc G, Davies L, Goodman D, Batalden P, Davidoff F, Stevens D. SQUIRE 2.0 (Standards for QUality Improvement Reporting Excellence): revised publication guidelines from a detailed consensus 
process. BMJ Qual Saf. 2016 Dec;25(12):986-92.

39. Joober R, Schmitz N, Annable L, Boksa P. Publication bias: what are the challenges and can they be overcome? J Psychiatry Neurosci. 2012 May;37(3):149-52.

40. Lyren A, Brilli R, Bird M, Lashutka N, Muething S. Ohio Children's Hospitals' Solutions for Patient Safety: A Framework for Pediatric Patient Safety Improvement. Journal for Healthcare Quality. 2016;38(4):213-22.

41. Solutions for Patient Safety. Operational Definitions [Internet]. Available from: https://www.solutionsforpatientsafety.org/wp-content/uploads/sps-operating-definitions.pdf.

\section{Tables}

Tables 1 and 2 can be found in the supplementary files section.

\section{Figures}

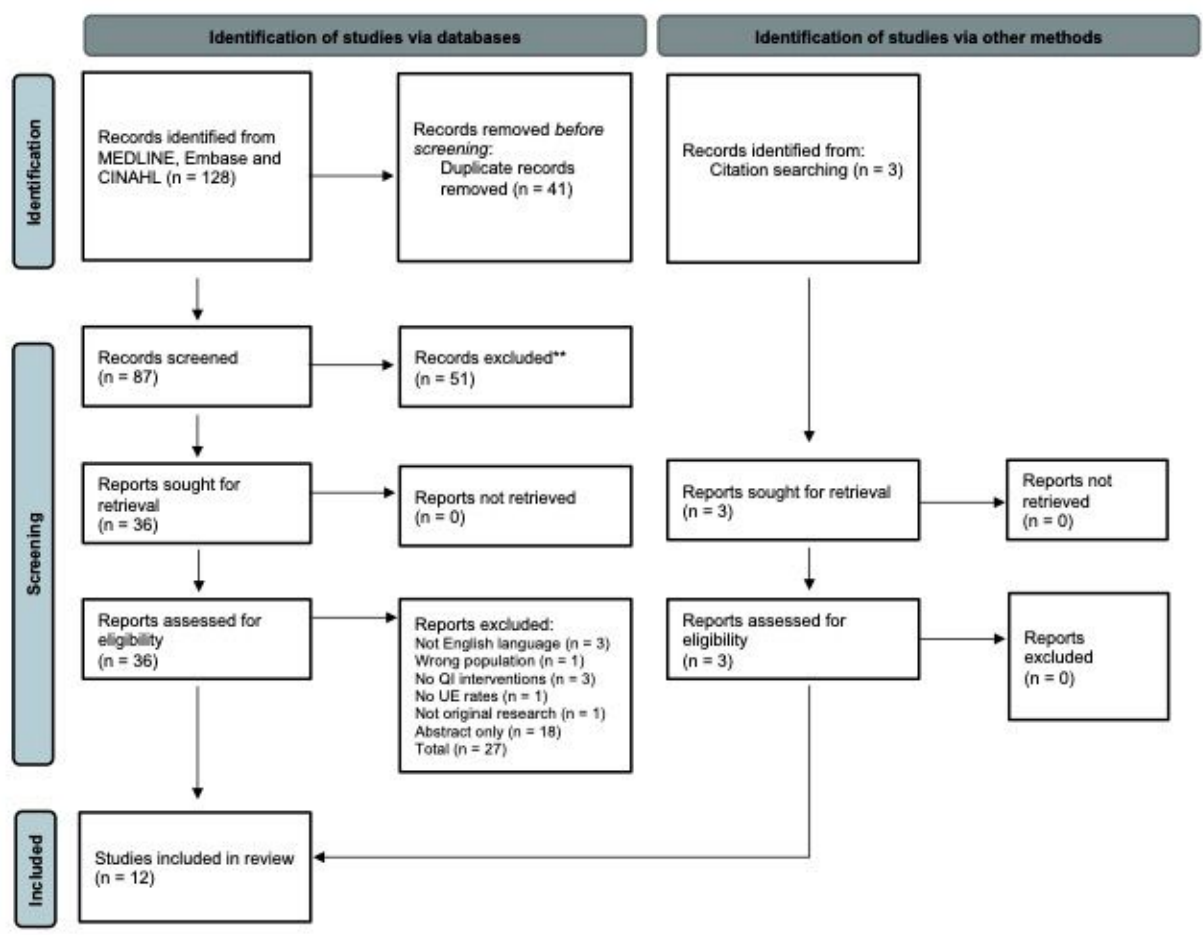

Figure 1

PRISMA 2020 Flow Diagram

Supplementary Files 
This is a list of supplementary files associated with this preprint. Click to download.

- Table1.docx

- STable1.docx

- STable2.docx

- STable3.docx

- Table2.docx

- STable4.docx 